\title{
Is Dutch Auction Suitable for Decomposable Tasks in Competitive Crowdsourcing Markets?
}

\author{
SANKAR KUMAR MRIDHA, INDIAN INSTITUTE OF ENGINEERING SCIENCE AND TECHNOL-
} OGY, SHIBPUR

MALAY BHATTACHARYYA, INDIAN INSTITUTE OF ENGINEERING SCIENCE AND TECHNOLOGY, SHIBPUR

\begin{abstract}
Competitive crowdsourcing is a popular strategic model for solving tasks in an efficient economical way. Competitive crowdsourcing platforms generally choose the winners for a single indivisible task following the conventional Dutch auction to minimize the cost. In this paper, we show that when the tasks are decomposable such mechanisms become biased for competitive crowdsourcing markets. As a solution to this, we suggest a variant of the Dutch auction (referred to as time-invariant Dutch auction) for solving decomposable tasks. Overall, some new insights about the mechanism design for competitive crowdsourcing markets are obtained.
\end{abstract}

\section{INTRODUCTION}

Internet has become a more successful medium to solve any kind of complex tasks as compared to the conventional offline modes. Online strategies are more profitable and powerful than the offline because they take limited time to find out a better solution involving a huge workforce. This has opened up the scope for new business models with a major economic impact. Crowdsourcing is such a popular business model where online jobs can be easily done over the Internet using the help of crowd in exchange of payments. The crowdsourcing models can be of two types - competitive and collaborative, which distinguishes the pattern of interaction between the crowd workers towards solving a problem. Understanding the behaviors of collaborative (Ipeirotis, 2010; Ross et al., 2010) and competitive crowdsourcing (Boudreau et al., 2011; Tang et al., 2011) environments has drawn immense attention in recent years.

The success of competitive crowdsourcing models mainly depends on the auction strategy it employs. Competitive bidding is an obvious strategy for both online and offline markets (Cramton, 2004). However, it might not be suitable for the environments with many changing factors. Crowd- 
sourcing is one such complex environment where diversity occurs frequently. Dutch auction is a well-known approach for managing various types of business environments although having some limitations (Milgrom, 1989; Bagwell, 1992). In this paper, we have tried to study the viability of using Dutch auction for competitive crowdsourcing markets where the tasks are decomposable. The concept of multiple winners, in an auction with combinatorial bids, for minimizing the possibility of collusion among the bidders has already been introduced (Kelly and Steinberg, 2000). We introduce the concept of time-invariant winner selection for better managing crowdsourcing markets.

In a competitive environment, we select the winners based on the best (say minimum) bid value. This selection can be time-variant (time of participation is given priority when there is a tie) or time-invariant. Now if the tasks can be further sub-divided, and the different parts are solved by multiple workers, then the environment becomes more competitive and the chance of getting a better bid value increases. For example, while finding the minimum cost multi-city itineraries through the crowdsourcing platforms like Flightfox (Bhattacharyya, 2013), different parts of the suggested itineraries can be combined together to obtain a better cost. Our motivation is to find out the best solution or true cost from the workers for a single task. The worker who has already posted the minimum bidding cost should also be forced through proper mechanism design for a better performance of the system (Narahari, 2014). We study these possibilities and suggest a suitable model for managing decomposable tasks in competitive crowdsourcing markets.

\section{MOTIVATION}

In this paper, we aim to better understand and control competitive crowdsourcing environments, where the tasks are decomposable. As an example, we can consider Flightfox, which is a crowdsourcing platform for finding out the best itineraries for a flyer. An older version of Flightfox was competitive but now it is collaborative. In the Flightfox platform, a requester (who posts the task as a contest) submits an itinerary (the contest and its details) and demands for the best flight (solution). All the workers (the experts who participate in the contest) compete to find out the best flight within the posted route (source and destination). The itineraries (contests) can be classified into three types - one-way, round-trip and multi-city. It is understandable that the task of finding multi-city flights are in fact decomposable.

A multi-city flight consists of multiple halts between the source and destination. Finding the best itinerary between multiple cities using Flightfox can be decomposed into the tasks of finding multiple one-way flights. For the multi-city contests, say between A and B, if a worker finds a flight between $A$ and $B$ via $C$ and another via $D$ then the total cost of the flight may vary and be lesser than the direct flights. Now, these intermediate flights can have varying costs. So, if the requester selects a combination of costs of decomposable itineraries form different workers then the outcome might be better than the total cost, that is selecting from a single worker. Similar such new platforms can also be developed where decomposable models might help to achieve a better work management through crowdsourcing to obtain a better solution.

\section{PRELIMINARIES AND DEFINITIONS}

Guided by the competitive crowdsourcing environments, we consider a distributed model for solving decomposable tasks where a requester $R$ posts a single task $T$ with (optionally) some given initial $\operatorname{cost} C \in Z^{+}$. A group of crowd workers $W=\left\{w_{1}, w_{2}, \ldots, w_{n}\right\}$ participate in an auction to bid for 


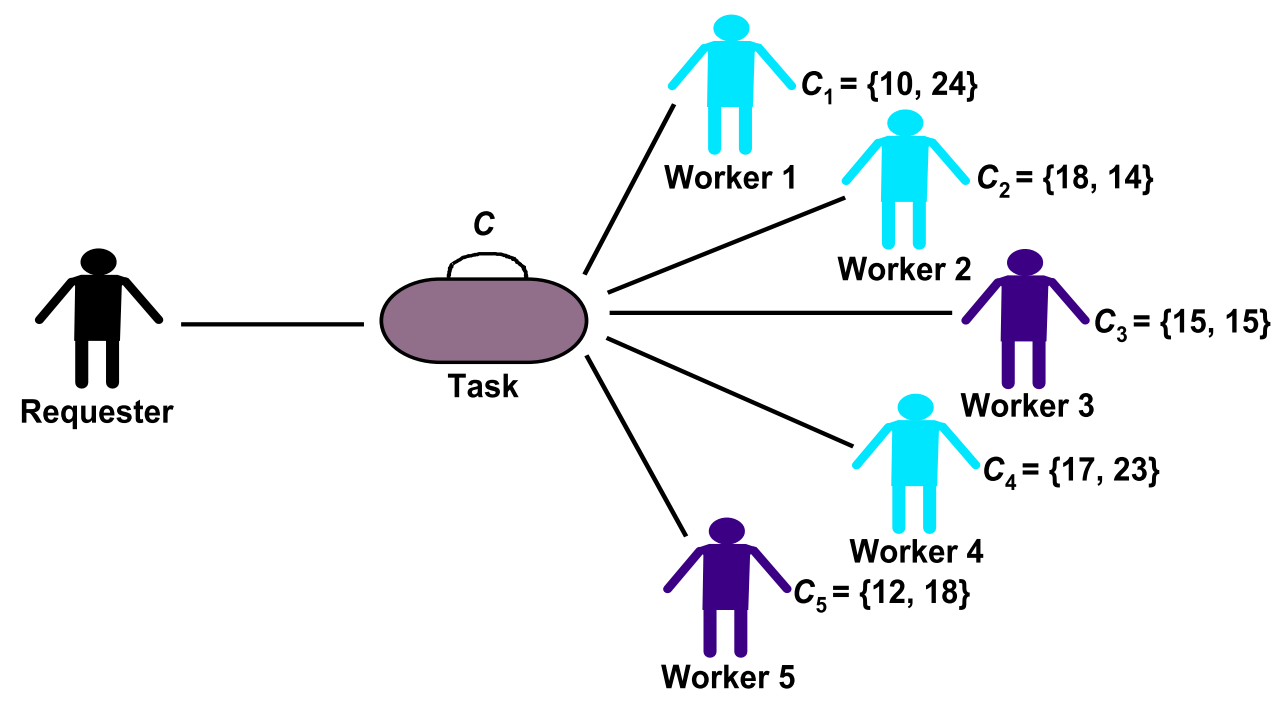

Figure 1. An example of competitive bidding with 2-decomposable tasks. Bidding cost of the $i^{\text {th }}$ worker is denoted as $C_{i}=\left\{C_{i}^{\prime}, C_{i}^{\prime \prime}\right\}$, where $C_{i}^{\prime}$ and $C_{i}^{\prime \prime}$ denote the costs for two different parts of the task. Multiple winners might exist (workers 3 and 5) for a single task if the workers do not collaborate. But if they collaborate on decomposable tasks it is possible to obtain an even better bidding cost (as the workers 1 and 2 become the joint winners here upon collaboration).

the task. We need to find out the best (minimum) bidding cost out of the independent bids. The crowd worker who is able to find out the best solution independently is selected as the winner. We additionally consider that the tasks are divisible into parts (and therefore into corresponding bids) and the crowd workers can collaborate to further choose a better solution. The model basically works in two phases. It does not take into account the level of information of each worker at the beginning and they are blindsided. The reason is to let them blindly compete for the best solution of the subtasks. When a worker is unaware about how better is the other solutions of the subtasks, he might give extra effort to find a better solution. As soon as he submits a solution he can see the other solutions to collaborate in the second phase.

Fig. 1 represents a scenario where bid values are raised for a single task by five crowd workers. Now, it can be seen that the tasks can be further sub-divided into independent parts and the workers can solve the task in combination. Generally, if the minimum bidding cost is posted by multiple workers, either both of them are selected as winners or the time of participation is considered. In Fig. 1, the workers 3 and 5 solve the task independently but the bidding costs remain the same, which is eventually the minimum. So, we can select both of them as the winners. Now, if we allow them to collaborate in the second phase, the joined bid values may get further reduced. In that case, the joint winners need not to include the current independent winners. Note that, if the workers 1 and 2 collaborate with each other then the joined bidding cost reduces than the current best single bids. On the other hand, the single minimum bidders can also collaborate to others (e.g., worker 2 collaborates with worker 5) to further reduce the cost. So, the strategy for selecting the winners for decomposable tasks can be of different types. We formalize these auction strategies hereunder. 
Definition 1(Time-variant Dutch auction). Time-variant Dutch auction refers to the open descending price auction in which the time of bidding is also given importance while selecting the winner.

Definition 2(Time-invariant Dutch auction). Time-invariant Dutch auction refers to the open descending price auction in which the time of bidding is not considered while selecting the winner.

Definition 3(Minimum time-variant bidder in Dutch auction). Given a set of bidders $\mathscr{W}=$ $\left\{w_{1}, w_{2}, \ldots, w_{n}\right\}$, participating in a time-variant Dutch auction with their corresponding bid values $\mathscr{C}=\left\{c_{1}, c_{2}, \ldots, c_{n}\right\}$, and the time of bidding $\mathscr{T}=\left\{t_{1}, t_{2}, \ldots, t_{n}\right\}$, respectively, the minimum timevariant bidder is denoted as $w_{m}$ such that $m=\arg _{i} \min _{i \in \arg _{j} \min _{j}} t_{i}$.

This definition basically depicts the earliest minimum bidder as the minimum time-variant bidder. We consider that the minimum time-variant bidder is the winner in time-variant Dutch auction. Note that, every task has a cost of completion and a bid value is associated with it. In our case, the bidders are the workers, bid values are the posted costs, and the tasks are nonempty decomposable. A nonempty decomposable task can be formally defined as follows.

Definition 4(K-nonempty decomposable task). A task is $K$-nonempty decomposable if it is divisible into $K$ nonempty subtasks that consume positive bid values.

By this definition, we simply neglect the possibility of dividing a task into multiple subtasks out of which one or more can be completed with a zero bid value. If the tasks are non-empty decomposable then it is possible for any two bidders to collaborate and the best combined bid value is considered as the joint bidding cost. E.g., in Fig. 1 if the workers 2 (with bidding cost $C_{2}=\left\{C_{2}^{\prime}, C_{2}^{\prime \prime}\right\}=\{18,14\}$ ) and 4 (with bidding cost $C_{4}=\left\{C_{4}^{\prime}, C_{4}^{\prime \prime}\right\}=\{17,23\}$ ) collaborate then their joint bidding cost will be $\min \left(C_{2}^{\prime}, C_{4}^{\prime}\right)+\min \left(C_{2}^{\prime \prime}, C_{4}^{\prime \prime}\right)=17+14=31$.

\section{THEORETICAL INSIGHTS}

After introducing the precursory details and formal definitions in the previous sections, we now present the following important result.

Lemma 1. The minimum bidder $w_{m}$ in a Dutch auction has the following winning probability if the bidders are allowed to collaborate and the tasks are 2-nonempty decomposable

$$
\mathscr{P}_{\text {win }}\left(w_{m}\right)\left\{\begin{array}{lr}
>\frac{1}{2}, & \text { if time-variant } \\
\leq \frac{1}{2}, & \text { if time-invariant }
\end{array}\right.
$$

Proof. The lemma comprises two cases of Dutch auction. To prove the first case, it is sufficient to establish that the winning probability of $w_{m}$ is more than $\frac{1}{2}$ in the worst case. This worst case arises when the closest two bidders collaborate on the decomposable task given. Let us assume that the minimum time-variant bid value (of the bidder $w_{m}$ in Dutch auction) is $c_{m}$. Suppose, the two closest bidders are $w_{m^{\prime}}$ and $w_{m^{\prime \prime}}$ and their bid values are $c_{m^{\prime}}$ and $c_{m^{\prime \prime}}$, respectively, such that $c_{m^{\prime}}=c_{m}+\delta^{\prime}$ and $c_{m^{\prime \prime}}=c_{m}+\delta^{\prime \prime}$ where $\delta^{\prime \prime} \geq \delta^{\prime} \geq 0$. As we are considering the minimum time-variant bidder as 
the winner, $w_{m^{\prime}}$ and $w_{m^{\prime \prime}}$ will jointly win if and only if they collaborate (considering that the tasks are 2-nonempty decomposable) and their combined bid value is strictly less than $c_{m}$. This is because they are time lagging. Therefore, the probability that $w_{m}$ will win even if $w_{m^{\prime}}$ and $w_{m^{\prime \prime}}$ collaborate is given by

$$
\mathscr{P}_{\text {win }}\left(w_{m}\right)=\frac{\Gamma\left(c_{m^{\prime} m^{\prime \prime}} \geq c_{m}\right)}{\Gamma\left(c_{m^{\prime} m^{\prime \prime}}\right)}
$$

where $\Gamma\left(c_{m^{\prime} m^{\prime \prime}}\right)$ denotes the number of possible combined bid values of $w_{m^{\prime}}$ and $w_{m^{\prime \prime}}$, and $\Gamma\left(c_{m^{\prime} m^{\prime \prime}}>\right.$ $c_{m}$ ) denotes the number of times it is more than or equal to $c_{m}$. For the brevity of proof, let us consider that the smallest indivisible unit of cost is $\psi>0$ (bid value is always positive because the task is nonempty decomposable). Then, we have $c_{m^{\prime} m^{\prime \prime}} \in\left[2 \psi,\left(c_{m^{\prime}}-\psi\right)+\left(c_{m^{\prime \prime}}-\psi\right)\right]$. By using this condition, we can rewrite Eqn. ((1)) as follows

$$
\begin{aligned}
\mathscr{P}_{\text {win }}\left(w_{m}\right) & =\frac{\frac{\left(c_{m^{\prime}}-\psi\right)+\left(c_{m^{\prime \prime}}-\psi\right)-c_{m}}{\psi}+1}{\frac{\left(c_{m^{\prime}}-\psi\right)+\left(c_{m^{\prime \prime}}-\psi\right)-2 \psi}{\psi}+1} \\
& =\frac{c_{m^{\prime}}+c_{m^{\prime \prime}}-c_{m}-\psi}{c_{m^{\prime}}+c_{m^{\prime \prime}}-3 \psi} \\
& =\frac{c_{m}+\delta^{\prime}+\delta^{\prime \prime}-\psi}{2 c_{m}+\delta^{\prime}+\delta^{\prime \prime}-3 \psi}\left[\because c_{m^{\prime}}=c_{m}+\delta^{\prime}, c_{m^{\prime \prime}}=c_{m}+\delta^{\prime \prime}\right] \\
& =\frac{c_{m}+\delta^{\prime}+\delta^{\prime \prime}-\psi}{2\left(c_{m}+\delta^{\prime}+\delta^{\prime \prime}-\psi\right)-\left(\delta^{\prime}+\delta^{\prime \prime}+\psi\right)} .
\end{aligned}
$$

Now, even if the closest two bidders in the worst case have bid values same as the minimum timevariant bidder (i.e., $\delta^{\prime}=\delta^{\prime \prime}=0$ ) and they collaborate, the resulting bid value is always positive ( $\psi>0$, because the tasks are nonempty decomposable). Therefore, $\delta^{\prime}+\delta^{\prime \prime}+\psi>0$ is always true. Hence the first case of the lemma.

Now, let us consider the second case. In time-invariant Dutch auction we allow multiple winners for the same bid value. So, in the worst case we have $\delta^{\prime}=\delta^{\prime \prime}=0$. Therefore, in the worst case $c_{m^{\prime}}=c_{m^{\prime \prime}}=c_{m}$. An interesting thing to note here is that for winning the bid in time-invariant case the minimum bidder has to be strictly better than the combined bids of others. By incorporating this idea and following the proof of the earlier case, we can derive the winning probability of minimum 
time-invariant bidder for 2-nonempty decomposable tasks as follows

$$
\begin{aligned}
\mathscr{P}_{\text {win }}\left(w_{m}\right)=\frac{\Gamma\left(c_{m^{\prime} m^{\prime \prime}}>c_{m}\right)}{\Gamma\left(c_{m^{\prime} m^{\prime \prime}}\right)} & =\frac{\frac{\left(c_{m}-\psi\right)+\left(c_{m}-\psi\right)-\left(c_{m}+\psi\right)}{\psi}+1}{\frac{\left(c_{m}-\psi\right)+\left(c_{m}-\psi\right)-2 \psi}{\psi}+1} \\
& =\frac{c_{m}-2 \psi}{2 c_{m}-3 \psi} \\
& =\frac{c_{m}-2 \psi}{2\left(c_{m}-2 \psi\right)+\psi} .
\end{aligned}
$$

Here the tasks being nonempty decomposable, we have $\psi \neq 0$, and thus $\mathscr{P}_{\text {win }}\left(w_{m}\right) \leq \frac{1}{2}$.

\section{THE SUGGESTED MODEL}

From Lemma 1, it is obvious that the minimum time-variant bidder in Dutch auction has a higher probability of winning the auction than the closest bidders (even if they collaborate). This makes the Dutch auction with decomposable tasks biased and provokes the minimum time-variant bidder not to collaborate with the others. As a solution to this, we now show that a strategy can be devised. If we consider that multiple winners can be selected based on the minimum bid value $\left(w_{m}\right)$, which turns the Dutch auction time-invariant, then it is clear (from the second case of Lemma 1) that the aforementioned bias can be removed. This will also force the minimum bidder to collaborate with others so that the chance of achieving a better bid value increases.

So, the strategy of selecting multiple winners based on time-invariant Dutch auction forces the current minimum bidder to further collaborate. This is because the risk of losing the bid is higher than winning it if the other competitors collaborate with each other (as highlighted in Lemma 1). This forces the minimum bidder also to collaborate (on decomposable tasks) constantly throughout the bidding process. It is interesting to discuss here the complexity incurred in the crowdsourcing model by decomposing the tasks into $k$ subtasks. Let there be $n$ number of workers participating in such a model. If the tasks are 2-nonempty decomposable (i.e., $k=2$ ) then each of them can submit at most two solutions (corresponding to the subtasks), and thus we have $O\left(n^{2}\right)$ possible solutions. If the tasks are nonempty decomposable for higher values of $k$ then this complexity will increase. However, it is evident that only a polynomial effect is incurred on the complexity of the model, and therefore it is scalable.

\section{CONCLUSION}

This paper theoretically analyzes the suitability of Dutch auction strategy for managing decomposable tasks in competitive crowdsourcing environments. Given a competitive environment, the scope of decomposing tasks introduces a collaborative advantage within the mechanism design. This provably guarantees to obtain reduced bid value for a posted task. Our study shows the limitation of time-invariant Dutch auction for the control of such environments. Based on this understanding, we suggest an appropriate model for a better (in terms of minimizing the cost) completion of decomposable tasks in a competitive crowdsourcing platform. 


\section{ACKNOWLEDGMENT}

The authors would like to thank Prof. Y. Narahari in the department of Computer Science and Automation of Indian Institute of Science for his important feedback over an initial version of this paper. The work of Malay Bhattacharyya is supported by the Visvesvaraya Young Faculty Research Fellowship 2015-16 of DeitY, Government of India.

\section{REFERENCES}

Bagwell, L. S. (1992). Dutch Auction Repurchases: An Analysis of Shareholder Heterogeneity. The Journal of Finance 47, 1 (1992), 71-105.

Bhattacharyya, M. (2013). Analyzing Flightfox: Who takes the Cake before the Take-off?. In Proceedings of the AAAI HCOMP Workshop on Crowdsourcing at Scale. Palm Springs, USA.

Boudreau, K. J, Lacetera, N, and Lakhani, K. L. (2011). Incentives and Problem Uncertainty in Innovation Contests: An Empirical Analysis. Management Science 57, 5 (2011), 843-863.

Cramton, P. (2004). Competitive Bidding Behavior in Uniform-Price Auction Markets. In Proceedings of the Hawaii International Conference on System Sciences. Big Island, HI, USA.

Ipeirotis, P. G. (2010). Analyzing the Amazon Mechanical Turk Marketplace. ACM XRDS 17, 2 (2010), 16-21.

Kelly, F and Steinberg, R. (2000). A Combinatorial Auction with Multiple Winners for Universal Service. Management Science 46, 4 (2000), 586-596.

Milgrom, P. (1989). Auctions and Bidding: A Primer. Journal of Economic Perspectives 3, 3 (1989), 3-22.

Narahari, Y. (2014). Game Theory and Mechanism Design. IISc Press and World Scientific.

Ross, J, Irani, L, Silberman, M. S, Zaldivar, A, and Tomlinson, B. (2010). Who are the crowdworkers?: shifting demographics in mechanical turk. In Proc. CHI EA 2010. ACM Press, Atlanta, Georgia, USA, 2863-2872.

Tang, J. C, Cebrian, M, Giacobe, N. A, Kim, H.-W, Kim, T, and Wickert, D. B. (2011). Reflecting on the DARPA Red Balloon Challenge. Commun. ACM 54, 4 (2011), 78. 